\title{
New opportunities for nanotechnology in the field of diagnostics based on proteomics
}

\author{
César Pascual García* and Sivashankar Krishnamoorthy
}

Nano-Enabled Medicine and Cosmetics group, Materials Research and Technology Department, Luxembourg Institute of Science and Technology (LIST), 41, rue du Brill, Belvaux, Luxembourg

\begin{abstract}
New developments in personalised medicine pose a technological challenge for the implementation of diagnostic devices demanding an increase for the speed, throughput and sensitivity for the detection of proteomic biomarkers. Following the tracks of genetics where the progress came through the analysis of DNA fragments, peptides, which are small fragments of proteins, can provide a new tool to get insights into the analysis of macromolecules. However, the current methods of combinatorial chemistry and sensing based on liquid handling and labelling, respectively, cannot cope with the demands of throughput and speed required by clinical applications. Nanotechnology holds promises to improve in-situ synthesis of microarrays and label free sensing to provide the combinatorial flexibility, quickness and sensitivity to help for the implementation of proteomic diagnosis in precision medicine.
\end{abstract}

\section{Introduction}

During the last decade, personalised medicines have progressed due to an unprecedented penetration of clinical devices for molecular diagnostics [1]. Most of these instruments like the next generation sequencers (NGS's) or point of care diagnosis (PoCdx) devices based on polymerase chain reaction (PCR) (like the Biofire Film Array, Genemark e-sensor, Luminex xTAG or Biocartis Idylla devices) operate in the field of genetics. At the base of this progress, there is an accurate manipulation of DNA. Outstanding examples of technology helping the decision makig of personalised diagnosis are the DNA sequencers developed by the former Ion Torrent instrument (currently Thermofisher) [2] or Oxford Nanopore [3], that demonstrated that electrochemical sensing can be used to deliver high-throughput quality data in a compact cost-efficient tool. Immunologist use now these kind of instruments combined with in-silico techniques to find alterations on the genes responsible of making proteins, driving developments that open the door to new therapies. The prospect of the post-genomic era is an improvement driven by proteomics. While genes can contain misleading structural of un-expressed information, proteomic biomarkers can provide more representative information of the cell activity revealing not only a more accurate diagnostic, but also an indication of the therapeutic drug by revealing the proteins that could be used in a possible vaccine [4].

However, the chemical control of proteins is not jet as good as the one of DNA, which hampers the progress of immunosensors for the implementation of proteomics at clinical level. Mass-spectrometry is nowadays the main tool for the analysis in research, but is not able to cope with the required amount of information needed for clinical decisions. In hospitals enzymes used in ELISA and antibodies for immunostaining provide some of the most sensitive and selective tests, but they are still generic tools not tune to the personal profile. Precision medicine is demanding a higher and more complex analysis of proteins at the hospital level. A possibility to address the required combinatorial diversity is to use smaller entities made by combination of the constituent amino acids, peptides. Peptides can provide a very rich variety of chemical combinations. With only 15 amino acids (a.a.'s) the number of possible combinations $\left(>10^{27}\right)$ is much bigger than the number of transistors shipped ever since the technology was invented $\left(>10^{21}\right)$. This diversity can only be addressed with flexible programmable molecular libraries, which are in the case of these molecules peptide microarrays. Peptide microarrays can hold in fact the potential to detect, differentiate and characterize proteins providing significant biological information [5,6].

In addition to the complexity and variability of proteins, protein diagnostics face the ultralow concentrations at which these molecules are found in biological conditions in the absence of molecular preamplification like in the case of DNA. Therefore, the assays have to employ ultra-sensitive transducers preferably with label free sensing. Nanostructured sensors open new perspectives since their size is comparable to the molecules to detect reaching good sensitivities with ultralow concentrations [7]. However few of these sensors are

*Correspondence to: García CP, Nano-Enabled Medicine and Cosmetics group, Materials Research and Technology Department, Luxembourg Institute of Science and Technology (LIST), 41, rue du Brill, Belvaux, Luxembour, E-mail: cesar.pascual@list.lu

Special Issue: Nanotechnology: Challenges and Perspectives in Medicine

Dr. Federica Valentini

Department of Sciences and Chemical Technologies

Tor Vergata University

Italy

Maurizio Talamo

Professor

Department of Enterprise Engineering Italy

Received: November 25, 2018; Accepted: December 20, 2018; Published: December 24, 2018 
candidates to be used for multiplexing the high number of spots present in peptide microarrays. Currently, plasmonic or highly sensitive field effect transistors (FETs) are the main candidates that can provide the miniaturisation necessary to offer hundreds of thousands of features per $\mathrm{cm}^{2}$. The biggest obstacle found by nanostructure nanosensors has been their standardisation that makes them reliable for clinical uses.

An improved chemical manipulation of proteomic entities combined with label free techniques is the request to implement proteomic diagnostics for personalised medicine. If the use of peptides is the chosen route, the challenge is producing high-throughput programmable peptide microarrays to study the possible interactions of proteins that can translate relevant clinical information. Currently peptide microarrays are still expensive and unreliable for their use in clinical diagnostics. They can benefit from the use of nanotechnologies to increase their yield and efficiency. As nanotechnologies are also rapidly advancing lowering the limits of detection of molecules in biological tissues and fluids now it seems possible that these tests will help in the decision making of clinicians in the next future. Here we review at glance the current technologies for the synthesis of microarrays and sensitive label free sensing in which nanotechnology can help to improve to provide the next generation diagnostic tools.

\section{Microarrays}

The implementation different strategic combinations of chemistries and microfluidic handling for in-situ synthesis using has increased the combinatorial potential solid phase peptide synthesis (Bruce Merrifield, Nobel prize for chemistry in 1984) by miniaturising the spots of peptides. Peptide microarrays currently commercially available are produced using three different approaches: liquid handling (SPOT microarrays produced by JPT [8]), printing techniques based on particle confined peptides (microarrays produced by PepPerPrint [9]), and optical deprotection of optical labile or acid labile groups [10]. SPOT is a technique based on the microfluidic dispensing of the reagents for insitu synthesis based on the deprotection of FMOC groups. They deliver up to 15 amino-acid (a.a.) microarrays using cellulosic substrates with a density of features around one spot $/ \mathrm{mm}$. Particle-based synthesis is based in the encapsulation of reagents on micro-particles and it is delivered and printed using the same techniques of inject printing. It also employs FMOC deprotection for the synthesis steps. The piezoelectric dispensers are able to reduce the size of the spots in virtually any kind of substrates, and the size of each spot is limited by the droplet/substrates interactions during the printing process. These methods have the disadvantage that their special resolution is limited by drop spreading and that they are sequential techniques, which makes that the synthesis time increases with the number of spots.

In 1991 by Fodor, et al. synthesised microarrays using optical deprotection of photo labile groups using masks similar to the ones employed in the fabrication of microelectronic devices [11]. The main advantage of optical deprotection is a real multiplexing of the synthesis since the reagents arrive simultaneously to all the cells, and the coupling is controlled by the addressable deprotection. In addition, optical in-situ synthesis also allowed to decrease the size of the peptide spots. Although in theory the limitation for the size is the wavelength diffraction limit, other practical effects related to fluidic fluctuations and set-up stability limit the size of each spot to several tenths of microns. With the introduction of digital mirror devices (DMDs) it was possible to increase the flexibility and automation of this synthesis, allowing a significant reduction of the costs, and different groups introduced different chemistries based also in FMOC (Shafer-N $[12,13]$ ), or acid labile groups like BOC (Intel CRP [14]). Optical deprotection groups yield average stepwise yields between 94 and $98 \%$ (total yield for a 15 -aa peptides between 40 and $73 \%$ ), but the real advantage is the number of features reaching easily densities of tens and hundreds of thousand features by $\mathrm{cm}^{2}$. Due to this high density of features it has been demonstrated applications like epitope mapping, and antibody detection $[15,16]$. Optical deprotection of acid-labile t-BOC groups using optically-generated acid provided total yields similar or better than traditional solid phase synthesis methods [14] close to $90 \%$ for 15 to 20 amino-acid synthesis.

An alternative approach for in-situ synthesis of biopolymers was introduced by Southern and Egeland [17]. The method achieved the deprotection of conventional BOC acid labile protecting groups by an electrochemically-generated acid during a redox reaction controlled by microelectrodes. An improvement was introduced by Combimatrix (currently Custom-Array) with the use of a porous substrate to slow down the proton diffusion [18], which allowed the commercialisation of a compact DNA microarray synthesiser, coupled to the detection of DNA FET with more than $96 \mathrm{~K}$ different positions (CustomArray, Inc., n.d.). However, when this platform was used to synthetize peptides, it did not reach sufficient acid control on each of the spots, providing a total yield of $\sim 70 \%$ on the deprotection of Trytyl-amino-acids (less than $0.5 \%$ of total yield for 15 -aa peptides). The demand to improve the synthesis of peptides is to improve the control over the acid in miniaturised cells. Recently we presented a microfluidic cell with an improved control of the acid in miniaturised dimensions, which can be used to improve the synthesis of biopolymers $[19,20]$.

\section{Sensing}

Field effect transistors (FETs) are the building blocks of the massively parallelised computer processors that have been miniaturised down to $15 \mathrm{~nm}$. A modification of these consisting in the ion sensitive field effect transistor (ISFET) is also the base for one of the bio-sensing schemes allowing multiplexing. In the ISFETs [21] the metal gate is replaced by an electrolyte connected to a reference electrode. The charged species binding to the surface change the gate potential to tune the conductivity in the channel of the transistor. The ISFET was a step forward in electrochemical sensing because semiconductors allow tuning the number of carriers to have a similar total charge than the one to be detected. The ISFET concept progressed with the decoration of the gate surface with selective molecules that were able to trap other charged analytes than inorganic ions, becoming sensitive to molecules like DNA and proteins [22]. Electrochemical immune-FETs are compatible with CMOS and when produced at high numbers they can be cost effective not only for high-end applications but also for point of care devices.

Nanotechnology has made bio-FETs much more sensitive because the size of the sensor became comparable to the electric field originated by the charged analytes thanks to the simultaneous control on the dimensions of the device and the number of carriers. An increasing number of materials are being introduced both in 1D (carbon nanotubes, semiconductor nanowires) and 2D (MoS2 or graphene) (for a recent review on immuno-FETs: [23]). Carbon-based materials and monolayer materials are very promising due to their high sensitivity, but they still hold many challenges regarding their manufacturability and integration. Semiconductor-based materials, on the other hand, can be fabricated with sub $50 \mathrm{~nm}$ dimensions with excellent control of the charge doping, using top-down fabrication strategies, both at prototype research and industry levels and without the need of particular 
modifications of standard procedures [24]. Silicon nanowire-field effect transistors (SiNW-FETs) are one of the candidates to be among the building blocks in the next future [25,26]. Their three-dimensional configuration makes them more efficient than planar FETs to detect ultra-low concentrations of molecules, due to a better gating [27-29] and because of efficient geometry in diffusion limited processes [7-9]. Proteins have been detected at very low quantities $[26,30]$ in different media including biological fluids [31-33] and tissues [34-36]. Owing to the well-known nanofabrication methods, and low operational current and voltages, SiNW-FETs can be easily integrated into CMOS chips where transducers and necessary circuits for signal processing are integrated on a single chip $[26,30,37,38]$. SiNW FETs bring promises of cost effective point of care (POC) and highly multiplexed sensors for personalised precision medicines in cancer therapies [39].

Regardless all their benefits, SiNW-FETs still have not been introduced into any clinical application, mainly due to their poor repeatability and reliability. Due to their small size, they are intrinsically sensitive to fabrication defects that propagate along the conduction channel, and the small total surface area increase the effect of thermodynamic fluctuations occurring during functionalisation. Current approaches are directed to improve the fabrication methods and the material reliability from the control of composition and homogeneity of the dielectric sensitive layer [40-42] and quality control on larger production batches $[28,43]$, but still there are fundamental challenges that require a global approach that include a detailed study of the bio-interactions in which the control of the adsorption of biomolecules represents a major challenge.

Plasmonic sensors represent also a main trend of nanostructured bio-sensors that allow multiplexing due to their miniaturisation potential and their large enhancement factors of optical signals. These devices use metal nanostructures to trap and enhance electromagnetic radiation close to surface. Such enhancement translates as higher sensitivity in detection of molecular analytes in different ways, including detection of changes to localized refractive index and enhancing fluorescence or vibrational Raman intensities. Amongst most prominent of these have been the surface plasmon resonance sensors, which is highly sensitive and label-free. Recently however, plasmonic sensors based on nanoparticles have emerged as a clear alternative owing to their simple, cost and space-saving configurations.

Amongst the nanoparticle based sensors, the Localized surface plasmon resonance (LSPR) sensors use changes to light intensity or wavelength to detect changes down to $<0.1 \mathrm{ng} / \mathrm{cm}^{2}$ concentrations of proteins [44,45]. By virtue of using refractive index to transduce the binding events, LSPR sensors are essentially label free. Surfaceenhanced Raman spectroscopy (SERS) based sensing or proteins offer also the advantage of label-free sensing, as they probe vibrational levels of the binding molecule which is unique to different molecules [46]. However, the best sensitivities achieved by use of SERS sensors has been achieved with molecules that have a high Raman cross-section, and as a result, different ways of achieving unique Raman labels using fluorescent labels became common [47-49]. While this delivered high sensitivities, it also rendered these approaches not label free. Despite the use of labels, the narrow vibrational Raman peaks enabled a high degree of spectral multiplexing, by enabling ease of resolving presence of a large population of molecules in a single spot [50]. Such spectral multiplexing, although can be performed also with fluorescence is limited to typically up to 4 , and further requires careful choice of molecules with well-separated fluorescence peaks. Surface-enhanced Fluorescence (SEF) [51] based plasmonic sensing has been shown to extend limits of an already sensitive fluorescence detection, pushing limits to femto-molar regime [52]. While this is not label-free, it is compatible with the existing fluorescence based assay configurations and could benefit from advances with fluorescent quantum dots in place of molecular fluorophores.

All the plasmonic sensors benefit from the use of noble metal structures, such as those of gold and silver as their plasmon resonances can be excited by visible light. All plasmonic sensors exhibit also a strong signal dependence on the distance of the reporter (the analytes themselves, or a secondary species that is further attached to the surfacebound analyte) of binding events from the sensing surface. This gives advantage of surface selectivity while also imposes a need to rationally design the optical/spectroscopic properties of the nanostructures and reliable fabrication approaches to maximize sensitivity. This often imposes the challenge for realization of plasmonic interfaces that can deliver high sensitivity, yet without compromise on quality and throughput, of which certain molecular self-assembly approaches have shown high promise [53-55]. Recent efforts have shown high promise in deploying plasmonic sensing in clinical diagnosis of cancer markers [56], high-throughput screening in microarrays [57,58], in-vitro as well as in-vivo deployment [52], intra-vesicular exosomal protein detection [59], and real-time structural analysis of protein conformational changes [60], as few examples.

\section{Conclusions}

Proteomics is the next step required for the progress of personalised medicines. While the understanding of the biological mechanisms have advanced, the clinical implementation is awaiting for the right tools for immune-diagnostics. The progress in the reliability of nanosensors together a compact integration with combinatorial methods implementing a flexible functionalisation has the potential to provide laboratories and hospitals the right tools for decision making of personalised treatments. Nanotechnology appears a necessary tool to improve both of the in-situ synthesis and sensing methods.

\section{Acknowledgements}

This work was financed by the Fonds National de la Recherche Luxembourg under projects NanopH (ATTRACT project 5718158) and PLASENS (C15/MS/10459961), respectively.

\section{References}

1. https://wwwweforumorg/agenda/2016/01/3-ways-personalized-medicine-will-affectyou/

2. Rothberg JM, Hinz W (2011) An integrated semiconductor device enabling non-optical genome sequencing. Nature 475: 348-352.

3. Jain M, Koren S, Miga KH, Quick J, Rand AC, et al. (2018) Nanopore sequencing and assembly of a human genome with ultra-long reads. Nat Biotechnol 36: 338-345. [Crossref]

4. https://wwwproteinatlasorg/humanproteome/cancer

5. Brennan DJ, O'Connor DP, Rexhepaj E, Ponten F, Gallagher WM (2010) Antibodybased proteomics: fast-tracking molecular diagnosis in oncology. Nat Rev Cancer 10: 605-617. [Crossref]

6. Grötzinger C (2016) Peptide microarrays for medical applications in autoimmunity, infection, and cancer. Methods Mol Biol 1352: 213-221.

7. Krishnamoorthy, S Nanostructured Sensors for Biomedical Applications-a Current Perspective. Curr Opin Biotechnol 2015, 34

8. Frank R (2002) The SPOT-synthesis technique Synthetic peptide arrays on membrane supports--principles and applications. J Immunol Methods 1: 13-26.

9. Stadler V, Felgenhauer T, Beyer M, Fernandez S, Leibe K, et al. (2008) Combinatorial synthesis of peptide arrays with a laser printer. Angew Chem Int Ed Engl 47: 71327135. [Crossref] 
10. Szymczak L, Kuo HY (2017) Peptide Arrays: Development and Application. Anal chem 90: 266-282.

11. Fodor SP, Read JR (1991) Light-directed, spatially addressable parallel chemical synthesis. Science 251: 767-773.

12. Hansen LB, Buus S, Schafer-Nielsen C (2013) Identification and Mapping of Linear Antibody Epitopes in Human Serum Albumin Using High-Density Peptide Arrays. PLOS One 8: e68902.

13. Shin DS, Lee KN, Yoo BW (2010) Automated Maskless Photolithography System for Peptide Microarray synthesis on a chip. J Comb Chem 12: 463-471.

14. Price JV (2012) On silico peptide microarrays for high-resolution mapping of antibody epitopes and diverse protein-protein interactions. Nature Med 18: 1434-1440.

15. Østerbye T (2015) Automated High-Throughput Mapping of Linear B-Cell Epitopes Using a Statistical Analysis of High-Density Peptide Microarray Data Methods. Mol Biol 215: 1348

16. Hansen CS (2017) ArrayPitope: Automated Analysis of Amino Acid Substitutions for Peptide Microarray-Based Antibody Epitope Mapping. PLoS One 12: e0168453.

17. Egeland RD, Southern EM (2005) Electrochemically directed synthesis of oligonucleotides for DNA microarray fabrication. Nucleic Acids Res 33: 125.

18. Maurer K (2006) Electrochemically Generated Acid and Its Containment to 100 Micron Reaction Areas for the Production of DNA Microarrays. PLOS one

19. Balakrishnan D, Lamblin G, Thomann JS, Berg AV, Olthuis W, et al. (2018) Electrochemical Control of $\mathrm{pH}$ in Nanoliter Volumes. Nano Lett 18: 2807-2815.

20. Balakrishnan D, Gerard M, Frari DD, Girod S, Olthuis W, et al. (2018) Redox Active Polymer as a pH Actuator on a Re-Sealable Microfluidic Platform. J Material Sci Eng 7: 3.

21. Bergbeld P (1970) Development of an ion-sensitive solid-state device for neurophysiological measurements. IEEE Trans Biomed Eng 1: 70-71.

22. Schöning, Michael J; Poghossian, Arshak (2002) Recent advances in biologically sensitive field-effect transistors (BioFETs). The Analyst 127: 1137-1151.

23. Kubota AC (2016) Recent Trends in Field-Effect Transistors-Based Immunosensors Chemosensors, $4(20)$

24. Penner RM (2012) Chemical sensing with nanowires. Annu Rev Anal Chem (Palo Alto Calif) 5: 461-485. [Crossref]

25. Cui Y, Wei Q, Park H, Lieber CM (2001) Nanowire nanosensors for highly sensitive and selective detection of biological and chemical species. Science 293: 1289-1292.

26. Stern (2007) Label-free immunodetection with CMOS-compatible semiconducting nanowires. Nature 445 .

27. Knopfmacher O, Tarasov A, Fu W, Wipf M, Niesen B, et al. (2010) Nernst limit in dualgated Si-nanowire FET sensors. Nano Lett 10: 2268-2274. [Crossref]

28. Rani D, Pachauri V, Mueller A, Vu XT, Nguyen TC, et al. (2016) On the use of scalable nanoISFET arrays of silicon with highly reproducible sensor performance for biosensor applications. ACS Omega 1: 84-92.

29. Rani D, Pachauri V, Ingebrandt S (2018) Silicon Nanowire Field-Effect Biosensors Label-Free Biosensing. Advanced Materials, Devices and Applications.

30. Pui TS, Agarwal A, Ye F, Huang Y, Chen P (2011) Nanoelectronic detection of triggered secretion of pro-inflammatory cytokines using CMOS compatible silicon nanowires. Biosens Bioelectron 26: 2746-2750.

31. Zhang GJ, Chai KTC, Luo HZH, Huang JM, Tay IGK, et al. (2012) Multiplexed detection of cardiac biomarkers in serum with nanowire arrays using readout ASIC Biosens Bioelectron 35: 218-223.

32. Stern E, Vacic A, Rajan NK, Criscione JM, Park J, et al. (2010) Label-free biomarker detection from whole blood. Nat Nanotechnol 5: 138-142. [Crossref]

33. Zhang YL, Chen RM, Xu L, Ning Y, Xie SG, et al. (2015) Silicon nanowire biosensor for highly sensitive and multiplexed detection of oral squamous cell carcinoma biomarkers in saliva. Anal Sci 31: 73-78.

34. Shehada N (2015) Ultrasensitive silicon nanowire for real-world gas sensing: noninvasive diagnosis of cancer from breath volatolome. Nano Lett 15: 1288-1295.

35. Dai X, Zhou W, Gao T, Liu J, Lieber CM (2016) Three-dimensional mapping and regulation of action potential propagation in nanoelectronics-innervated tissues. Nat Nanotechnol 11: 776-782

36. Puppo F (2016) SiNW-FET in-air biosensors for high sensitive and specific detection in breast tumor extract. IEEE Sens $J$ 16: 3374-3381.

37. Gao A, Lu N, Dai P, Li T, Pei H, et al. (2011) Silicon-Nanowire-Based CMOSCompatible Field Effect Transistor Nanosensors for Ultrasensitive Electrical Detection of Nucleic Acids. Nano Lett 11: 3974-3978.
38. Lei KM, Mak PI, Law MK, Martins RP (2016) CMOS biosensors for in vitro diagnosis - transducing mechanisms and applications. Lab Chip 16: 3664-3681. [Crossref]

39. Doucey MA, Carrara S (2018) Nanowire Sensors in Cancer. Trends Biotechnol [Crossref]

40. Noor MO, Krull UJ (2014) Silicon nanowires as field-effect transducers for biosensor development: A review. Analytica Chimica Acta 825: 1-25.

41. Luye M, Ye C, Sawtelle SD, Wipf M, Xuexin D et al. (2015) Silicon Nanowire FieldEffect Transistors: A Versatile Class of Potentiometric Nanobiosensors. Access IEEE 3: $287-302$.

42. Zafar S, D’Emic C, Jagtiani A, Kratschmer E, Miao X, et al. (2018) Silicon Nanowire Field Effect Transistor Sensors with Minimal Sensor-to-Sensor Variations and Enhanced Sensing Characteristics. ACS Nano 12: 6577-6587. [Crossref]

43. Ishikawa FN, Curreli C, Chang HK, Chen PC, Zhang R, et al. (2009) Calibration Method for Nanowire Biosensors to Suppress Device-to-Device Variation. ACS Nano 3: 3969-3976.

44. Dahlin AB, Tegenfeldt JO, Höök F (2006) Improving the instrumental resolution of sensors based on localized surface plasmon resonance. Anal Chem 78: 4416-4423. [Crossref]

45. Anker JN, Hall WP, Lyandres O, Shah NC, Zhao J, et al. (2008) Biosensing with plasmonic nanosensors. Nat Mater 7: 442-453. [Crossref]

46. Han XX, Zhao B, Ozaki Y (2009) Surface-enhanced Raman scattering for protein detection. Anal Bioanal Chem 394: 1719-1727. [Crossref]

47. Han XX, Zhao B, Ozaki Y (2009) Surface-enhanced Raman scattering for protein detection. Anal Bioanal Chem 394: 1719-1727. [Crossref]

48. Halas NJ, Moskovits M (2013) Surface-Enhanced Raman Spectroscopy: Substrates and Materials for Research and Applications. MRS Bull 38: 607-611.

49. Stiles PL, Dieringer JA, Shah NC, Van Duyne RP (2008) Surface-enhanced Raman spectroscopy. Annu Rev Anal Chem (Palo Alto Calif) 1: 601-626. [Crossref]

50. Stevenson R, Faulds K, Graham D (2010) Quantitative DNA Analysis Using SurfaceEnhanced Resonance Raman Scattering. Surf Enhanc Raman Spectrosc 241-262.

51. Lakowicz JR, Geddes CD, Gryczynski I, Malicka J, Gryczynski Z, et al. (2004) Advances in surface-enhanced fluorescence. J Fluoresc 14: 425-441. [Crossref]

52. Zhang B, Kumar RB, Dai H, Feldman BJ (2014) A plasmonic chip for biomarker discovery and diagnosis of type 1 diabetes. Nat Med 20: 948-953. [Crossref]

53. Yap FL, Thoniyot P, Krishnan S, Krishnamoorthy S (2012) Nanoparticle Cluster Arrays for High-Performance SERS through Directed Self-Assembly on Flat Substrates and on Optical Fibers. ACS Nano 6.

54. Dinda S, Suresh V, Thoniyot P, Balcytis A, Juodkazis S, et al. (2015) Engineering 3D Nanoplasmonic Assemblies for High Performance Spectroscopic Sensing. ACS Appl Mater Interfaces 7.

55. Krishnamoorthy S, Krishnan S, Thoniyot P, Low HY (2011) Inherently Reproducible Fabrication of Plasmonic Nanoparticle Arrays for SERS by Combining Nanoimprint and Copolymer Lithography. ACS Appl Mater Interfaces 3.

56. Li M, Cushing SK, Zhang J, Suri S, Evans R, et al. (2013) Three-Dimensional Hierarchical Plasmonic Nano-Architecture Enhanced Surface-Enhanced Raman Scattering Immunosensor for Cancer Biomarker Detection in Blood Plasma. ACS Nano 7: 4967-4976.

57. Tabakman SM, Lau L, Robinson JT, Price J, Sherlock SP, et al. (2011) Plasmonic Substrates for Multiplexed Protein Microarrays with Femtomolar Sensitivity and Broad Dynamic Range. Nat Commun 2: 466.

58. Cetin AE, Coskun AF, Galarreta BC, Huang M, Herman D, et al. (2014) Handheld High-Throughput Plasmonic Biosensor Using Computational on-Chip Imaging Light. Sci Appl 3: e122.

59. Park J, Im H, Hong S (2018) Analyses of Intravesicular Exosomal Proteins Using a Nano-Plasmonic System. ACS Photonics 5: 487-494. [Crossref]

60. Etezadi D, Warner JB, Lashuel HA, Altug H (2018) Real-Time In Situ Secondary Structure Analysis of Protein Monolayer with Mid-Infrared Plasmonic. Nanoantennas ACS Sensors 3: 1109-1117.

Copyright: C2018 García CP. This is an open-access article distributed under the terms of the Creative Commons Attribution License, which permits unrestricted use, distribution, and reproduction in any medium, provided the original author and source are credited. 